\title{
Qualitative And Quantitative Detection of Surgical Pathogenic Microorganisms Escherichia Coli and Staphylococcus Aureus Based on ddPCR System
}

\section{Tiantian Zhang}

First Hospital of Shanxi Medical University

\section{Zhiqiang Niu}

Shanxi Medical University

\section{Feng Wu}

Shanxi Medical University

\section{Zongkun Chen}

Shanxi Medical University

Jun xu

First Hospital of Shanxi Medical University

\section{Kewei Jiang}

Peking University People's Hospital

Zhiyong Lai ( $\nabla 09774722 @ q q . c o m)$

First Hospital of Shanxi Medical University

\section{Research Article}

Keywords: Droplet digital PCR, Escherichia coli, Staphylococcus aureus, Blood samples

Posted Date: December 10th, 2020

DOl: https://doi.org/10.21203/rs.3.rs-121224/v1

License: (c) (i) This work is licensed under a Creative Commons Attribution 4.0 International License.

Read Full License

Version of Record: A version of this preprint was published at Scientific Reports on April 22nd, 2021. See the published version at https://doi.org/10.1038/s41598-021-87824-5. 


\section{Abstract}

Bacterial culture and drug susceptibility testing are used to identify bacteria associated with infections. Nevertheless, the process requires several days from collection to the identification of bacterial species and drug resistance patterns. The digital PCR system is a rapidly developing quantitative detection technology widely used in many fields, including pathogenic microorganism detection, early diagnosis of tumor markers, and analysis of gene expression with its advantages. The purpose of this study was to use a droplet digital PCR system to identify bacteria in blood samples, to explore its ability to identify common pathogenic microorganisms. We designed primers and probes for Escherichia coli and Staphylococcus aureus specific genes for the ddPCR system to identify in blood samples mixed with both organisms. The system had extremely high detection accuracy in samples and the detection rate of E. coli was $13.1-21.4 \%$ and that of S. aureus was $50-88.3 \%$. The system identified blood samples containing both bacteria, with detection rates of $18.1 \%-97 \%$. The ddPCR system qualitatively and quantitatively measured common pathogenic microorganisms in blood samples with high sensitivity and accuracy, providing rapid and accurate detection of pathogenic microorganisms.

\section{Introduction}

Despite economic development, with health systems in various countries worldwide continuously improving, infectious diseases are still one of the main threats to human health. The 2018 Global Burden of Disease Study reported that 7.069 million people died from infectious diseases worldwide, accounting for $12.6 \%$ of the global deaths, and the mortality rate was $97 / 100000^{1}$. In this context, infectious diseases remain a major public health problem.

Early diagnosis and rational application of antibiotics are essential for significance for management of infectious diseases. At present, clinical use of antibiotics depends on empirical treatment. In addition, the over-use of antibiotics not only increases the resistance of bacteria and drug-induced diseases, but also consumes substantial health care resources ${ }^{2}$. A study reported that the annual cost of treatment for infectious diseases is about $\$ 1.5-2$ billion ${ }^{3}$. One of the reasons for this phenomenon is that the pathogenic microorganisms cannot be quickly and accurately identified in the early stage of infections, often leading to irrational use of antibiotics and other medical treatment measures.

The most common pathogenic bacteria in departments of general surgery are Escherichia coli, Staphylococcus aureus, Pseudomonas aeruginosa, Klebsiella pneumoniae, and Acinetobacter baumannii 4 . Traditional bacterial detection and identification methods are based on bacterial culture and biochemical identification, and testing usually requires several days to one week. The diagnosis of bacterial infectious diseases often relies on the specific detection of pathogenic bacteria as the gold standard, and in the process of clinical diagnosis and treatment, the use of antibiotics must be based on the bacterial culture results and drug sensitivity test results of biological samples. However, due to factors such as detection time, bacterial culture growth characteristics, non-pathogenic bacteria contamination, and improper material selection, the test results in actual work may generate false negatives or false 
positives. Incorrect identification results not only in delayed treatment, wasting medical resources, but in mistaken diagnosis and treatment. For these reasons, rapid accurate identification of pathogenic microorganisms has become an intense focus of research.

In recent years, molecular biology techniques have been applied for the detection of pathogenic microorganisms, and have attracted attention due to their high specificity, lower time-requirement and reduced incidence of cross-infection. These techniques include immune response ${ }^{2}$, polymerase chain reaction ${ }^{5}$ and gene chip ${ }^{6}$. Since its advent, PCR technology has been widely used in many research fields because of its simple, intuitive, economical, and rapid detection characteristics ${ }^{7}$. In the course of the continuous development of molecular biology technology in the context of a variety of detection needs, several new PCR technologies have emerged. In 1992, Higuchi first proposed real-time quantitative polymerase chain reaction technology ${ }^{8}$. First, qPCR technology amplifies target nucleic acid fragments by polymerase chain reaction based on specific nucleic acid sequences of pathogenic microorganisms. Then, fluorescent signals are generated and accumulated during the amplification process of the target nucleic acid fragment. Finally, standard curves are generated to identify and quantify the target strain. However, because the amplification efficiency of common qPCR technology is limited, it is difficult to accurately quantify the original template using $\mathrm{Ct}$ values.

Digital polymerase chain reaction was first proposed by Kenneth Kinzler and Bert Vogelstein in 1999. This is a PCR technology that truly achieves absolute quantification after qPCR technology. Digital PCR is divided into microfluidic digital PCR, droplet digital PCR (ddPCR), and chip digital PCR depending on the method of liquid separation? ${ }^{9}$.

The working principle of ddPCR system is as follows: First, the reaction system containing nucleic acid molecules is subjected to extreme dilution and dispersed through a droplet generator into a reaction unit that can be as small as a pico-upgrade. Each reaction unit then acts as an independent PCR reactor, with or without target molecules (DNA or RNA) to be detected. After PCR amplification is completed, the signal of each reaction unit is measured, and the presence or absence of the end point signal is used as a criterion (the droplet with a fluorescent signal is interpreted as "1", and the droplet without a fluorescent signal is interpreted as "0"). Finally, the concentration or copy number of the target molecule to be detected is calculated according to the Poisson distribution principle. Compared with traditional qPCR, digital PCR has higher sensitivity, specificity, and accuracy, and plays an important role in many fields, including early diagnosis of tumor markers ${ }^{10}$, gene expression product analysis ${ }^{11}$, food safety testing ${ }^{12}$, pathogenic microorganism testing ${ }^{13}$, common genetic disease testing, and non-invasive prenatal diagnosis $^{14}$.

In the present study, we used real-time fluorescent quantitative PCR and droplet digital PCR to test the advantages and the reliability for the identification of simulated bacteremia blood samples using droplet digital PCR in biological sample testing, so as to provide the theoretical basis for subsequent clinical sample testing. 


\section{Results}

\section{Identification of bacterial strains using the ddPCR system}

The ddPCR system identification various concentrations of E. coli ATCC25922 nucleic acid (Fig. 1A, corresponding to $\mathrm{A} 05$ to $\mathrm{H} 05$ ). The reasons for the positive results in the negative control group may be that the ddPCR system has an extremely high detection sensitivity, together with the aerosol formed by droplets of bacterial nucleic acid that spreads in the air. The nucleic acid then contaminated the reaction system of the negative control group. The actual copy number of bacteria from A05 to E05 measured using the ddPCR system was analyzed using the theoretical value and $R^{2}=0.9995$ (Fig. 1A Table 1).

Table 1

Dynamic range of the ddPCR-based assay. Detecting the E. coli ATCC25922 nucleic acid template by ddPCR system

\begin{tabular}{|llllll|}
\hline Counts of bacterial nucleic acid template & $\mathbf{5 6 0} \mathbf{~ p g}$ & $\mathbf{5 6} \mathbf{~ p g}$ & $\mathbf{5 . 6} \mathbf{~ p g}$ & $\mathbf{1 . 4} \mathbf{~ p g}$ & $\mathbf{3 5 0} \mathbf{~ g}$ \\
\hline Theoretical copy number & 130000 & 13000 & 1300 & 130 & 32.5 \\
\hline Logarithmic transformation & 5.1139 & 4.1139 & 3.1139 & 2.1139 & 1.5119 \\
\hline Actual number of copies & 135800 & 13220 & 1134 & 120 & 34 \\
\hline Logarithmic transformation & 5.1329 & 4.1212 & 3.0546 & 2.0792 & 1.5315 \\
\hline
\end{tabular}

The ddPCR system identified various concentrations of $S$. aureus ATCC25923 nucleic acid (Fig. 1B, corresponding to $\mathrm{A} 01$ to $\mathrm{H} 01$ ), and the measured value of bacterial nucleic acid was very close to the theoretical value. The actual copy number of bacteria from A01 to C01 measured using the ddPCR system was analyzed using the theoretical value and $R^{2}=1$ (Fig. 1B, Table 2).

Table 2

Dynamic range of the ddPCR-based assay. Detecting the Staphylococcus aureus ATCC25923 nucleic acid template by ddPCR system

\begin{tabular}{|llll|}
\hline Counts of bacterial nucleic acid template & $\mathbf{3 1} \mathbf{~ p g}$ & $\mathbf{3 . 1} \mathbf{~ p g}$ & $\mathbf{3 1 0} \mathbf{f g}$ \\
\hline Theoretical copy number & 13000 & 1300 & 130 \\
\hline Logarithmic transformation & 4.1139 & 3.1139 & 2.1139 \\
\hline Actual number of copies & 11120 & 1058 & 96 \\
\hline Logarithmic transformation & 4.0461 & 3.0245 & 1.9823 \\
\hline
\end{tabular}

\section{The Ddpcr System Simultaneously Identified Two Bacterial Strains}


In the two sets of experiments, the actual DNA copy numbers of ATCC25922 and ATCC25923 were very close to the theoretical values, and there was almost no interference between them. The detection rate of bacteria was as high as $80 \%$ or even $90 \%$ (Fig. 2 ,Tables 3 and 4).

Table 3

Quantitative results of the ddPCR system simultaneously detecting two bacterial strains (bacteria counts of E. coli:bacteria counts of Staphylococcus aureus $=10: 1$ )

\begin{tabular}{|lll|}
\hline Bacterial strain & ATCC25922 & ATCC25923 \\
\hline Theoretical value & 6500 & 650 \\
\hline Measured value & 5420 & 572 \\
\hline Extraction rate(\%) & 83.4 & 88 \\
\hline
\end{tabular}

Table 4

Quantitative results of the ddPCR system simultaneously detecting two bacterial strains (bacteria counts of E. coli:bacteria counts of Staphylococcus aureus $=1: 10$ )

\begin{tabular}{|lll|}
\hline Bacterial strain & ATCC25922 & ATCC25923 \\
\hline Theoretical value & 650 & 6500 \\
\hline Measured value & 494 & 6000 \\
\hline Extraction rate(\%) & 76 & 92.3 \\
\hline
\end{tabular}

\section{Bacteria Count}

We added E. coli and S. aureus to $50 \mathrm{ml}$ of Luria broth and placed it in an orbital shaker at $37^{\circ} \mathrm{C}$ overnight. We then diluted the bacterial solutions $10^{6}$ times and performed a rolling ball scratching board. We calculated the number of colonies in the culture medium and found that the number of colonies of ATCC25922 was $10^{7}$, and the number of colonies of S. aureus ATCC25923 was 70 (Fig. 3). The concentration of the $E$. coli ATCC25922 bacterial solution was $1.25 \times 10^{9}$ cells $/ \mathrm{ml}$, and the concentration of $S$. aureus ATCC25923 bacterial solution was $6.7 \times 10^{8} \mathrm{cells} / \mathrm{ml}$. Before the experiment, we diluted $E$. coli and $S$. aureus solutions in a gradient.

\section{Detection Of Simulated Bacteria Using The Ddpcr System}

We used the ddPCR system to identify bacterial nucleic acid extracted from the simulated bacteremia blood samples. We counted the number of bacteria by counting the number of nucleic acid templates. The number of bacteria detected using the ddPCR system and the theoretical number of bacteria are 
listed in the Tables 5 and 6 . We took the average number of bacteria detected in the three groups and calculated the logarithm of the average number of bacteria to perform fitting analysis (Fig. 4, ATCC25922: $\mathrm{R}^{2}=0.9998$, ATCC25923: $\left.\mathrm{R}^{2}=0.9996\right)$. The $E$. coli ATCC25922 nucleic acid extraction rate in simulated bacteria was $13.1-21.4 \%$, and the $S$. aureus ATCC25923 nucleic acid extraction rate in simulated bacteria was $50-88.3 \%$.

Table 5

Detecting E. coli ATCC25922 nucleic acid extracted from the simulated bacteremia blood sample

\begin{tabular}{|llllll|}
\hline Counts of bacteria & $\mathbf{1 0 4 0 0 0 0}$ & $\mathbf{1 0 4 0 0 0}$ & $\mathbf{1 0 4 0 0}$ & $\mathbf{1 0 4 0}$ & $\mathbf{1 0 4}$ \\
\hline Parallel group 1 & 136400 & 16460 & 1924 & 188 & 16.8 \\
\hline Parallel group 2 & 137400 & 15680 & 1740 & 174 & 24 \\
\hline Parallel group 3 & 135800 & 15300 & 1704 & 192 & 26 \\
\hline Average & 136533.3 & 15813.3 & 1789.3 & 184.7 & 22.3 \\
\hline
\end{tabular}

Table 6

Detecting Staphylococcus aureus ATCC25923 nucleic acid extracted from the simulated bacteremia blood sample

\begin{tabular}{|lllllll|}
\hline Counts of bacteria & $\mathbf{1 0 4 0 0 0}$ & $\mathbf{1 0 4 0 0}$ & $\mathbf{1 0 4 0}$ & $\mathbf{1 0 4}$ & $\mathbf{5 0}$ & $\mathbf{2 5}$ \\
\hline Parallel group 1 & 88800 & 7000 & 730 & 62 & 20 & 12.8 \\
\hline Parallel group 2 & 92400 & 7320 & 694 & 72 & 28 & 11 \\
\hline Parallel group 3 & 94400 & 7200 & 706 & 62 & 32 & 13.6 \\
\hline Average & 91866.7 & 7173.3 & 710 & 65.3 & 26.7 & 12.5 \\
\hline
\end{tabular}

We used the ddPCR system to measure bacterial nucleic acid extracted from the simulated bacteremia blood samples contaminated by E. coli and $S$. aureus simultaneously (Tables 7 and 8 ). We took logarithm of the actually measured number of bacteria to make a fitting curve (Fig. 5, ATCC25922: $R^{2}=0.9532$, ATCC25923: $\left.\mathrm{R}^{2}=0.9958\right)$. The bacterial nucleic acid extraction rate of $E$. coli ATCC25922 was $18.1-77 \%$, and that of S. aureus ATCC25923 was $44.9-97 \%$. 
Table 7

Detecting E. coli ATCC25922 nucleic acid extracted from simulated bacteremia blood samples contaminated by Escherichia coli and Staphylococcus aureus

\begin{tabular}{|llllll|}
\hline Counts of bacteria & 104000 & 10400 & 1040 & 104 & 10.4 \\
\hline Parallel group 1 & 20180 & 1960 & 242 & 36 & 10 \\
\hline Parallel group 2 & 21100 & 1960 & 248 & 14 & 6 \\
\hline Parallel group 3 & 20440 & 1740 & 258 & 16 & 8 \\
\hline Average & 20573 & 1887 & 249 & 22 & 8 \\
\hline
\end{tabular}

Table 8

Detecting Staphylococcus aureus ATCC25923 nucleic acid extracted from simulated bacteremia blood samples contaminated by Escherichia coli and Staphylococcus aureus

\begin{tabular}{|llllll|}
\hline Counts of bacteria & $\mathbf{1 0 4 0 0 0}$ & $\mathbf{1 0 4 0 0}$ & $\mathbf{1 0 4 0}$ & $\mathbf{1 0 4}$ & $\mathbf{1 0 . 4}$ \\
\hline Parallel group 1 & 89000 & 8380 & 932 & 116 & 10 \\
\hline Parallel group 2 & 87400 & 8580 & 1010 & 106 & 0 \\
\hline Parallel group 3 & 85600 & 8160 & 1026 & 80 & 4 \\
\hline Average & 87333 & 8373 & 989 & 101 & 5 \\
\hline
\end{tabular}

\section{Discussion}

We found that the sensitivity and accuracy of the ddPCR system have obvious advantages compared with real-time fluorescent quantitative PCR. The ddPCR system retains high sensitivity and accuracy when simultaneously detecting two pathogenic microorganisms. In addition, the ddPCR system performs absolute quantification without need for a standard curve. However, the detection rate of bacteria in bacterial blood samples using the ddPCR system is lower than that of purified bacteria samples. The reason for this may be that when bacterial nucleic acid is extracted from bacterial blood samples, human blood, EDTA in the anticoagulation tube, and the elution and enrichment of nucleic acid all have a negative impact on the extraction of bacterial nucleic acid. Even so, the rate of extracting bacterial nucleic acid from bacterial blood samples by the ddPCR system was between $15 \%$ and $80 \%$.

We found that, when the concentration of bacteria in the blood was greater than $10 \mathrm{cell} / \mathrm{sl}$, the ddPCR system detected bacteria stably. At present, clinical laboratories determine whether bacteremia is present based on the blood culture instruments reporting positive and the reporting time is about 6-18 hours. Generally speaking, it takes about 3 days from the collection of blood samples to the completion of bacterial culture and antibiotic sensitivity testing. If we design unique probes and primers for specific bacterial and drug-resistance genes, the ddPCR system can identify bacterial species and drug resistance gene simultaneously, with detection times less than 4 hours. Therefore, our research suggests that the 
ddPCR technical platform is superior for identification of pathogenic microorganisms. The ddPCR system accurately and effectively detect pathogenic microorganisms, allowing for more targeted antibiotic therapy and minimizing misuse of antibiotics, drug toxicity, and drug resistance, all which could provide a sufficient theoretical basis for the preventive use of antibiotics and could reduce economic burdens on patients.

Currently, the coronavirus (COVID-19) epidemic is spreading all over the world. The pathogenic microorganism of this pneumonia has been identified as a new coronavirus (Severe Acute Respiratory Syndrome Coronavirus 2, SARS-CoV-2). In acute respiratory infections, reverse transcription polymerase chain reaction is usually used to detect pathogenic viruses from respiratory secretions in nucleic acid testing, and is the "gold standard" for diagnosis of related cases ${ }^{15}$. However, the RT-PCR test result can also produce false negatives ${ }^{16}$. The reasons are the time of specimen collection, the quality or type of specimen, the transportation of the specimen, the ability of the virus to mutate, and the low sensitivity of RT-PCR and polymerase chain reaction inhibition ${ }^{17-19}$. Therefore, an efficient, fast, and accurate laboratory diagnosis technology is the key to ensure early diagnosis, timely treatment, and prevent the development of the epidemic. Based on the its advantages, digital PCR provides a new, efficient, and accurate detection method for SARS-CoV-2 detection.

\section{Methods}

\section{Bacterial strains, experimental reagents and instruments}

The bacterial strains used were provided by the Laboratory of Peking University People's Hospital (Table S1), and the reagents and instruments used were provided by the laboratory of Tsinghua University (Tables S2 and S3).

\section{Extraction of bacterial nucleic acid}

The bacterial strains were propagated and cultured in $100 \mathrm{ml}$ Luria broth . Nucleic acids were extracted from the four bacteria using the QIAamp cador Pathogen Mini Kit (QIAGEN, Germany) according to the methods suggested in manufacturer's instructions. We used Nanodrop to measure the extracted nucleic acid concentrations and stored samples at $-20^{\circ} \mathrm{C}$.

\section{Design of primers and probes}

The specific genes of bacterial strains were selected according to the GenBank database. The specific genes of E. coli and S. aureus were SWG-9 and coagulase COA gene, respectively. Primers and probes were designed for conserved regions in SWG-9 and coagulase COA gene sequences (Table S4 and S5). We used Oligo 7.0 and Primer Express 3.0 software to design and evaluate primers and probes for the nucleic acid sequence containing the site to be detected. The sequences were delivered to Thermo Fisher Scientific $(\mathrm{CN})$ to synthesize the primers and probes. The 5 'end of the $E$. coli probe was labeled with 6FAM fluorescent molecule, the 5 ' end of the $S$. aureus probe was labeled with VIC fluorescent molecule. 


\section{Workflow of the ddPCR system}

We carried out ddPCR using the QX200 ddPCR system (Bio-Rad, CA) according to the manufacturer's instructions. The QX200 ddPCR system (Bio-Rad, CA) consists of four steps: (1) preparation of the reaction mixture using a ddPCR amplification volume $20 \mu \mathrm{L}$ (Table S6); (2) droplet generation; (3) ddPCR amplification (Table S7); and (4) droplet reading and analysis of results using QuantaSoft 3.0 software.

\section{Detection of bacterial strains using a real-time quantitative PCR system}

The SYBR Green real-time quantitative PCR system and the MGB probe real-time quantitative PCR system were used to quantify the gradient-diluted nucleic acid of E. coli standard strain (ATCC25922) to determine the sensitivity range of the two real-time quantitative PCR. The template dilution gradient (copies/ $\mu \mathrm{L}$ ) of ATCC25922 was $10^{6}, 10^{5}, 10^{4}, 10^{3}, 10^{2}, 10^{1}, 1$, and 0 . Each group of experiments was repeated three times in parallel.

\section{Detection of bacterial strains by ddPCR system}

We used the ddPCR system to quantify the gradient-diluted nucleic acid of E. coli (ATCC25922) to determine the sensitivity range of the ddPCR system. The template dilution gradient (copies/20 $\mu$ l) of ATCC25922 were $10^{5}, 10^{4}, 10^{3}, 10^{2}, 25,1.5625$, and 0 . Each group of experiments was repeated three times in parallel.

\section{The ddPCR system simultaneously detects two bacterial strains}

Various numbers of DNA templates, specific primers and probes (ATCC25922: 5'-FAM/3'MGB, ATCC25923: 5'-VIC/3'MGB) of the two bacterial strains were added to a ddPCR reaction system (Group A, bacteria counts of $E$. coli: bacteria counts of $S$. aureus $=10: 1$, Group B, bacteria counts of $E$. coli: bacteria counts of $S$. aureus $=1: 10$ ). We counted DNA template numbers of $E$. coli and $S$. aureus, and calculated the detection rate of the two bacteria.

\section{Detection of blood samples of simulated bacteremia blood sample using the ddPCR system}

The blood samples were obtained from the Department of Gastroenterological Surgery, Peking University People's Hospital. All of the blood samples donors included in this study provided their informed consent. The study was approved by the Human Ethics Committee of the Peking University People's Hospital (China). All methods were carried out in "accordance" with the approved guidelines and regulations. The blood collection tube were pretreated with EDTA anticoagulation. Three simulations of bacteremic blood samples were prepared, Group A: $100 \mu \mathrm{L}$ of the serially-diluted suspensions of E. coli (ATCC25922) were injected into $400 \mu \mathrm{L}$ of blood treated with EDTA. Group B: $100 \mu \mathrm{L}$ of the serially diluted suspension of the S. aureus strain (ATCC25923) was injected into the $400 \mu \mathrm{L}$ blood treated with EDTA. Group C: $100 \mu \mathrm{L}$ of the ATCC25922 and ATCC25923 suspensions were diluted into gradients, then added to the blood samples with $400 \mu \mathrm{L}$ EDTA. Extraction of nucleic acid from bacterial blood specimens was performed as follows: nucleic acid from bacterial blood specimens was extracted using a bacterial nucleic acid kit 
(Qiagen, Germany) We used $50 \mu$ l of solution buffer to elute DNA from the column and store at $80^{\circ} \mathrm{C}$. The extracted nucleic acid was detected using the ddPCR system. Repeat the test three times in parallel.

\section{Statistical analysis}

The detection results were statistically analyzed using SPSS 22.0, GraphPad Prism 5 and OriginPro 2016 software, and the linear dynamic range of the method was measured by $R^{2}$ value.

\section{Declarations}

\section{Acknowledgements}

We thank members of our laboratory for helpful discussion and Professor Guo Yong of Tsinghua University, Co for the support of bioinformatics analysis.

\section{Author contributions:}

ZhiyongLai, Kewei Jiang, Tiantian Zhang, Feng Wu, Zongkun Chen, Jun Xu and Zhiqiang Niu conceived and designed the study. Tiantian Zhang and Zhiqiang Niu wrote the manuscript. Zhiyong Lai and Kewei Jiang reviewed and edited the manuscript. All authors reviewed the manuscript.

\section{Competing interests}

The authors declare that they have no competing interests.

\section{References}

1. Global, Regional, and National Age-Sex-Specific Mortality for 282 Causes of Death in 195 Countries and Territories, 1980-2017: A Systematic Analysis for the Global Burden of Disease Study 2017. Lancet. 392, 1736-1788 (2018).

2. Alekseeva, L. P. et al. [Comparative Assessment of Dot-Immunoassay and Immunochromatography Methods for Detection of 01 Serogroup of Vibrio Cholerae]. Zh Mikrobiol Epidemiol Immunobiol. 8893 (2010).

3. Sun, B. Nosocomial Infection in China: Management Status and Solutions. Am. J. Infect. Control. 44, 851-852 (2016).

4. Shan, K. et al. Evaluation of Resistance by Clinically Pathogenic Bacteria to Antimicrobials and Common Disinfectants in Beijing, China. J. Nippon Med. Sch. 85, 302-308 (2018).

5. Blais, B. W. \& Martinez-Perez, A. A Simple PCR-based Macroarray System for Detection of Multiple Gene Markers in the Identification of Priority Enterohemorrhagic Escherichia Coli. J Food Prot. 74, 365-372 (2011).

6. Herbert, M. A. et al. Genetic Islands of Streptococcus Agalactiae Strains NEM316 and 2603VR and their Presence in Other Group B Streptococcal Strains. BMC Microbiol. 5, 31 (2005). 
7. Saiki, R. K. et al. Enzymatic Amplification of Beta-Globin Genomic Sequences and Restriction Site Analysis for Diagnosis of Sickle Cell Anemia. Science. 230, 1350-1354 (1985).

8. Higuchi, R., Dollinger, G., Walsh, P. S. \& Griffith, R. Simultaneous Amplification and Detection of Specific DNA Sequences. Biotechnology (N Y). 10, 413-417 (1992).

9. Vogelstein, B. \& Kinzler, K. W. Digital PCR. Proc Natl Acad Sci U S A. 96, 9236-9241 (1999).

10. Oehler, V. G. et al. Absolute Quantitative Detection of ABL Tyrosine Kinase Domain Point Mutations in Chronic Myeloid Leukemia Using a Novel Nanofluidic Platform and Mutation-Specific PCR. Leukemia. 23, 396-399 (2009).

11. White, A. K. et al. High-Throughput Microfluidic Single-Cell RT-qPCR. Proc Natl Acad Sci U SA. 108, 13999-14004 (2011).

12. Corbisier, P., Bhat, S., Partis, L., Xie, V. R. \& Emslie, K. R. Absolute Quantification of Genetically Modified MON810 Maize (Zea Mays L.) by Digital Polymerase Chain Reaction. Anal. Bioanal. Chem. 396, 2143-2150 (2010).

13. Strain, M. C. et al. Highly Precise Measurement of HIV DNA by Droplet Digital PCR. PLoS One. 8, e55943 (2013).

14. Lee, S. Y. et al. A New Approach of Digital PCR System for Non-Invasive Prenatal Screening of Trisomy 21. Clin. Chim. Acta. 476, 75-80 (2018).

15. Xu, Y. et al. Significance of Serology Testing to Assist Timely Diagnosis of SARS-CoV-2 Infections: Implication From a Family Cluster. Emerg Microbes Infect. 9, 924-927 (2020).

16. Sethuraman, N., Jeremiah, S. S. \& Ryo, A. Interpreting Diagnostic Tests for SARS-CoV-2. JAMA. 323, 2249-2251 (2020).

17. Kucirka, L. M., Lauer, S. A., Laeyendecker, O., Boon, D. \& Lessler, J. Variation in False-Negative Rate of Reverse Transcriptase Polymerase Chain Reaction-Based SARS-CoV-2 Tests by Time Since Exposure. Ann. Intern. Med. 173, 262-267 (2020).

18. Caruana, G. et al. Diagnostic Strategies for SARS-CoV-2 Infection and Interpretation of Microbiological Results. Clin Microbiol Infect. (2020).

19. Schrader, C., Schielke, A., Ellerbroek, L. \& Johne, R. PCR Inhibitors - Occurrence, Properties and Removal. J. Appl. Microbiol. 113, 1014-1026 (2012).

\section{Figures}




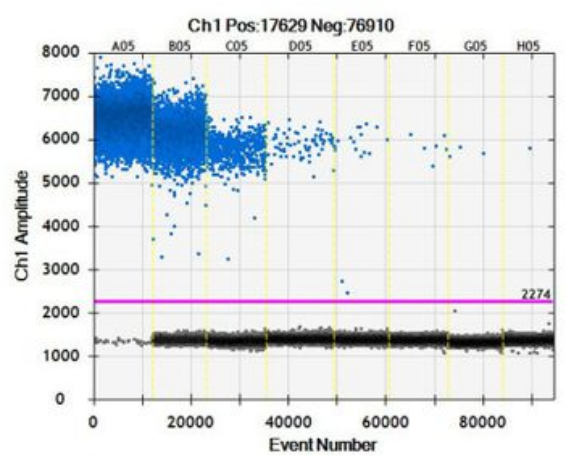

A

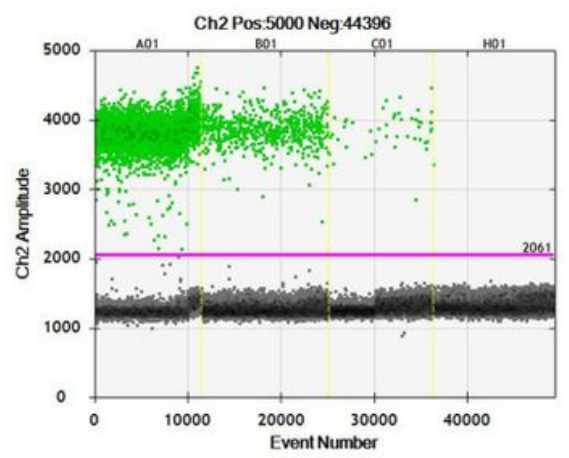

B
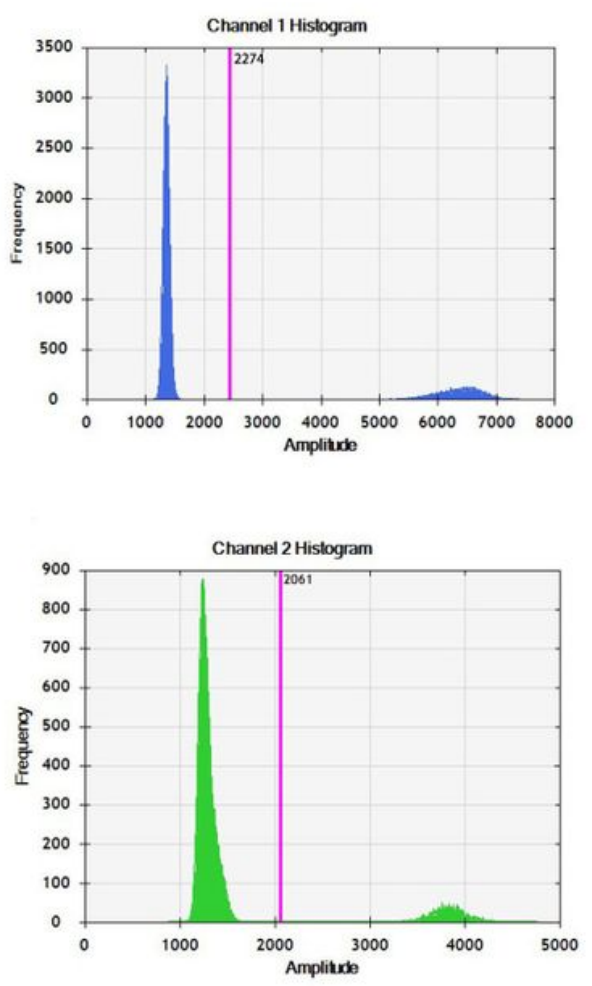
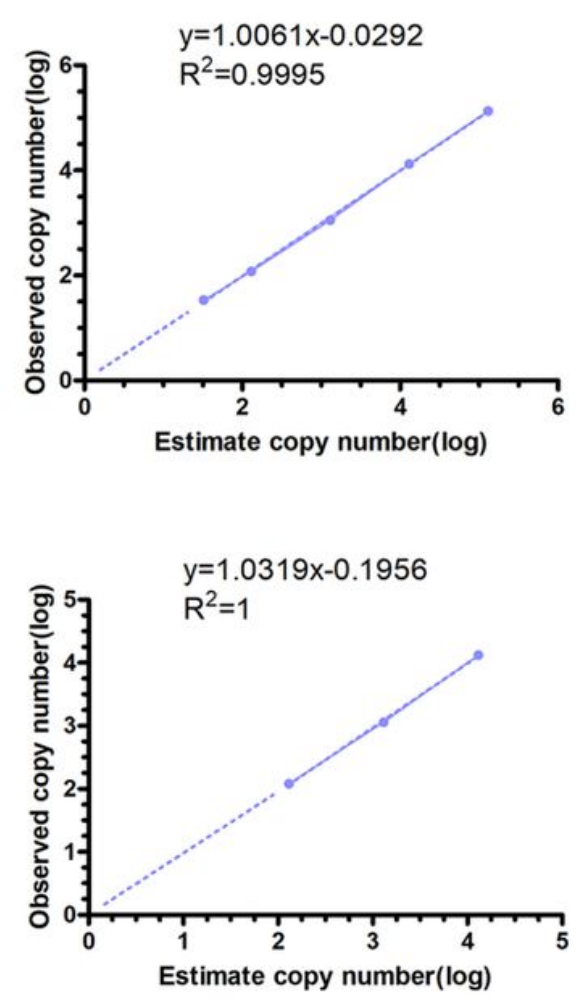

Figure 1

Dynamic range of the ddPCR-based assay. Detecting the bacterial nucleic acid by ddPCR system, A: The 1D droplet spots of FAM fluorescence for E. coli ATCC25922 were shown on the left and middle. B: The 1D droplet spots of VIC fluorescence for Staphylococcus aureus ATCC25923 were shown on the left and middle The estimated copy numbers of E.coli ATCC25922 nucleic acid template (copies/20 $\mu$ l) is $1.3 \times 105$, $1.3 \times 104,1.3 \times 103,1.3 \times 102,32,8,2$, and NC. The estimated copy numbers of Staphylococcus aureus

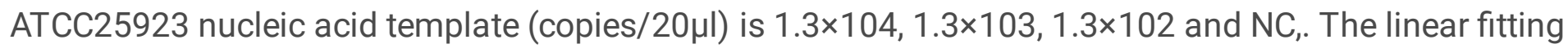
curves of Escherichia coli ATCC25922 and Staphylococcus aureus ATCC25923 were shown on the right. 


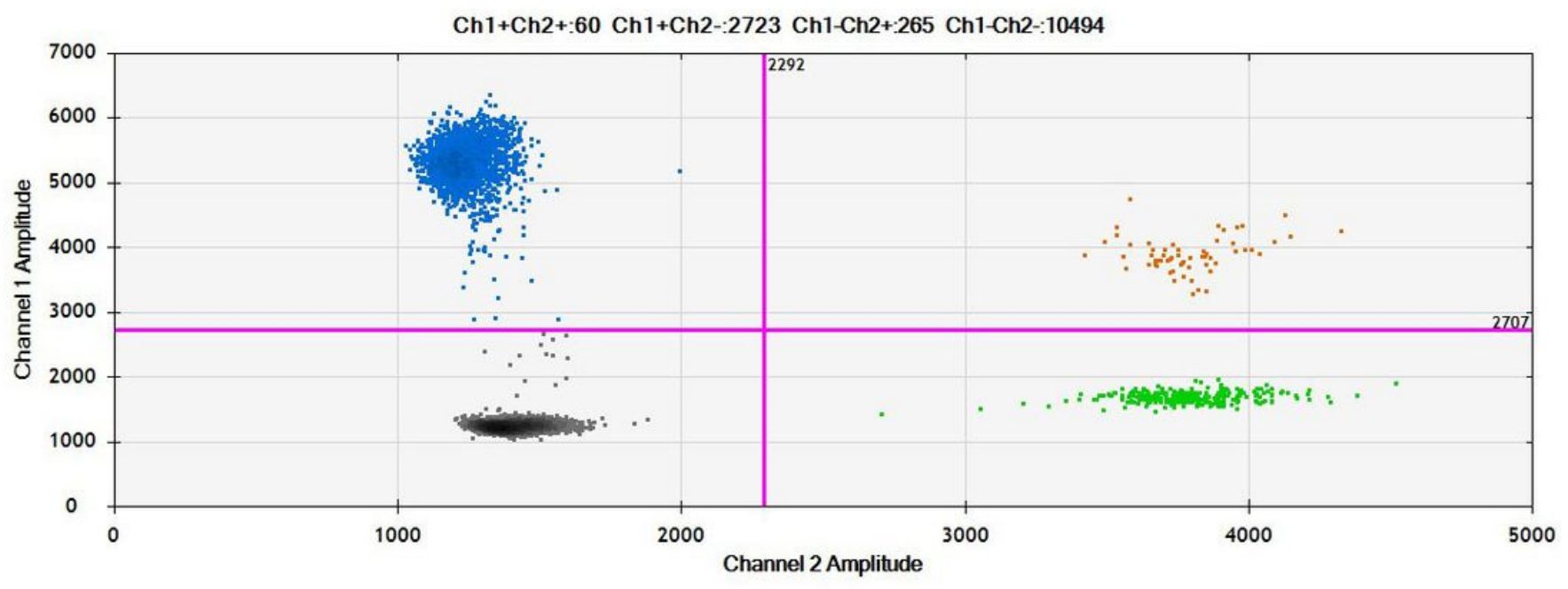

A

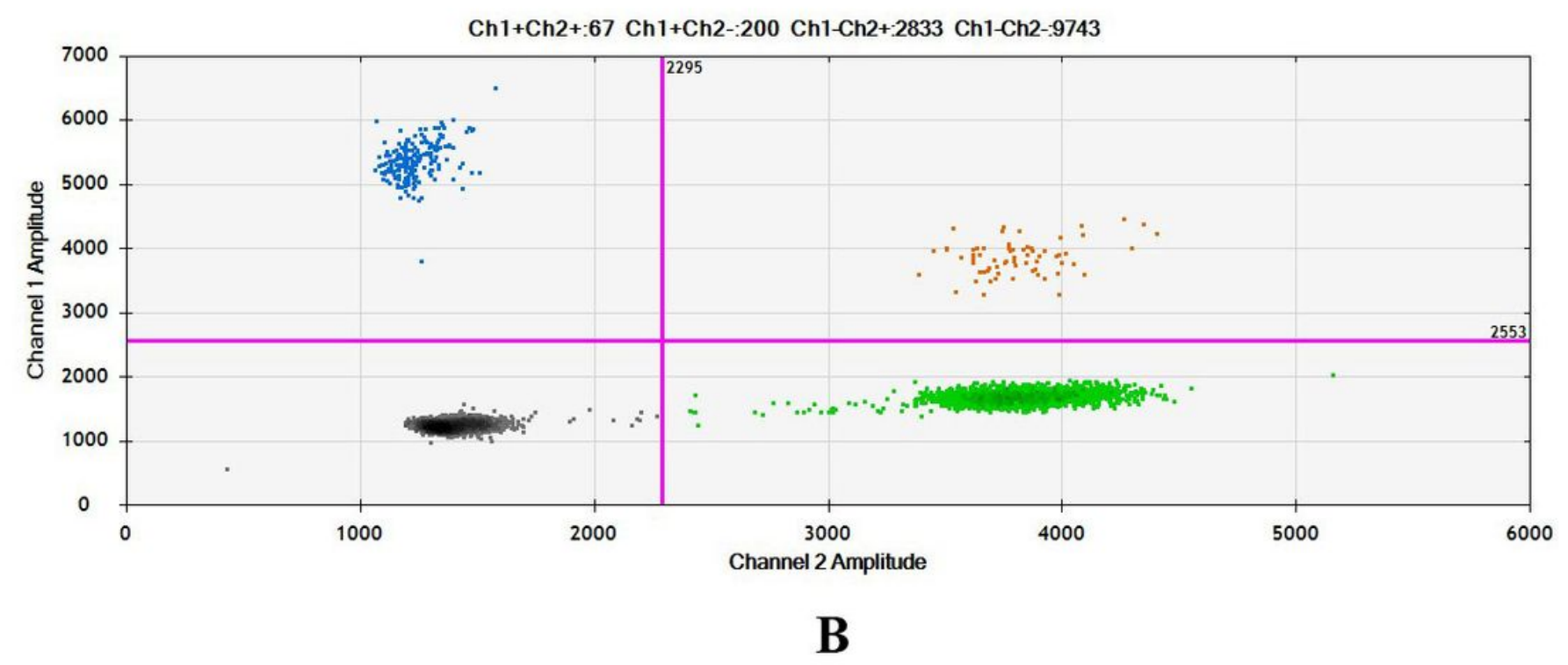

Figure 2

Quantitative results of the ddPCR system simultaneously detecting two bacterial strains. The 2D drop plots of Escherichia coli ATCC25922 and Staphylococcus aureus ATCC25923 by ddPCR system. A: bacteria counts of E. coli:bacteria counts of Staphylococcus aureus=10:1, B: bacteria counts of E. coli:bacteria counts of Staphylococcus aureus=1:10. 


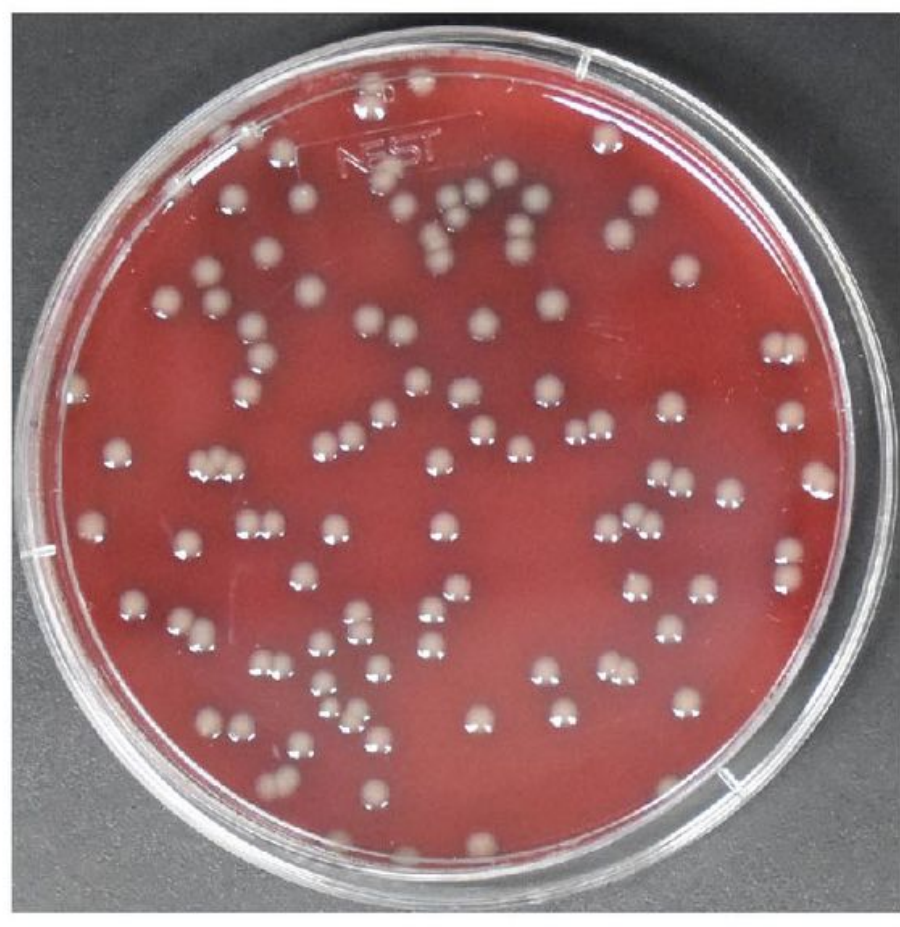

A

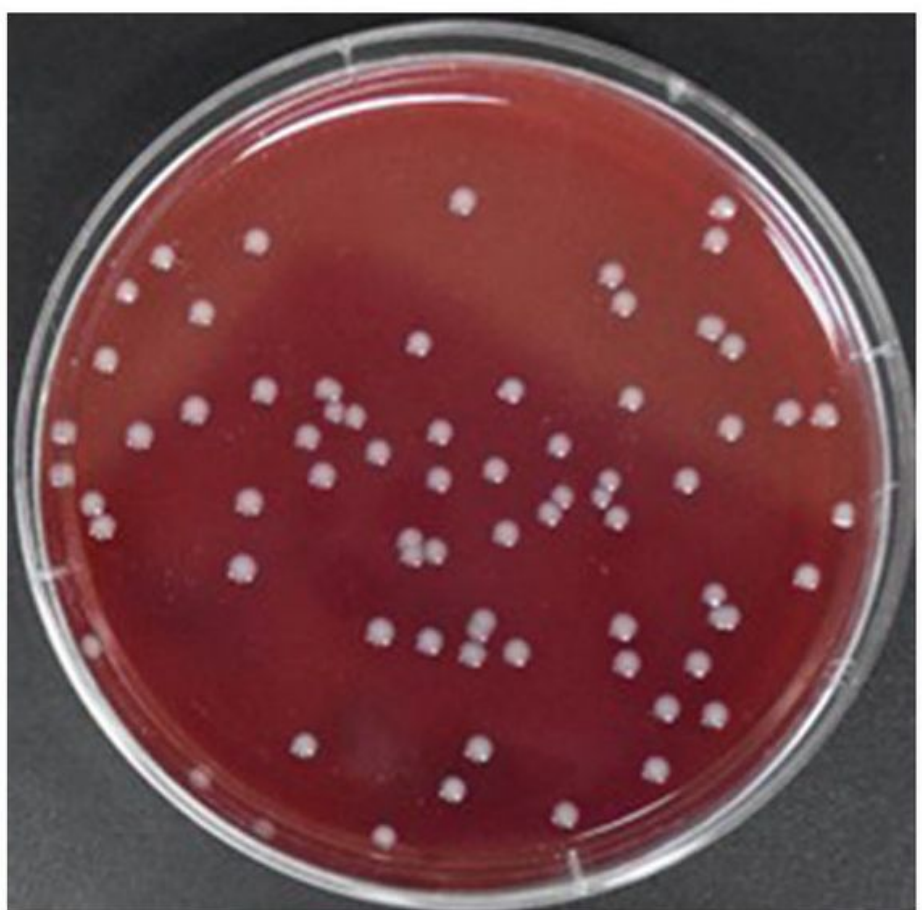

B

\section{Figure 3}

Bacterial culture and bacterial count. A: colony number of Escherichia coli ATCC25922 is 107, B: colony number of Staphylococcus aureus ATCC25923 is 70. 


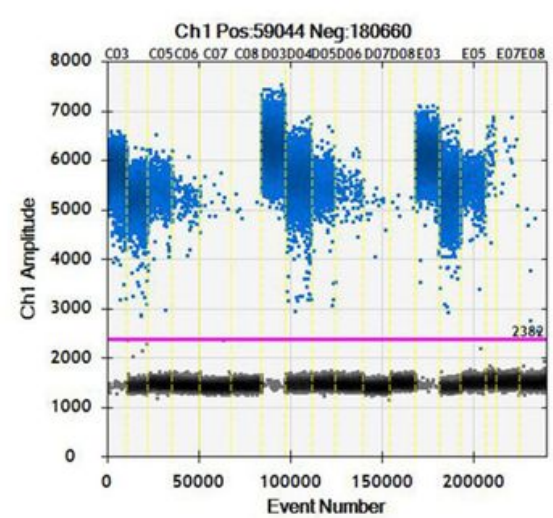

A

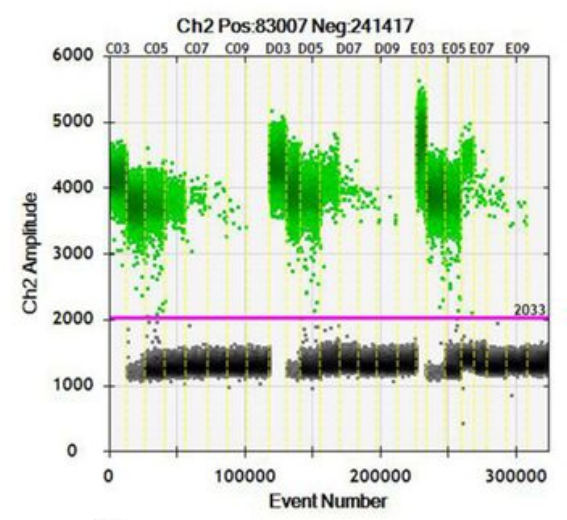

B
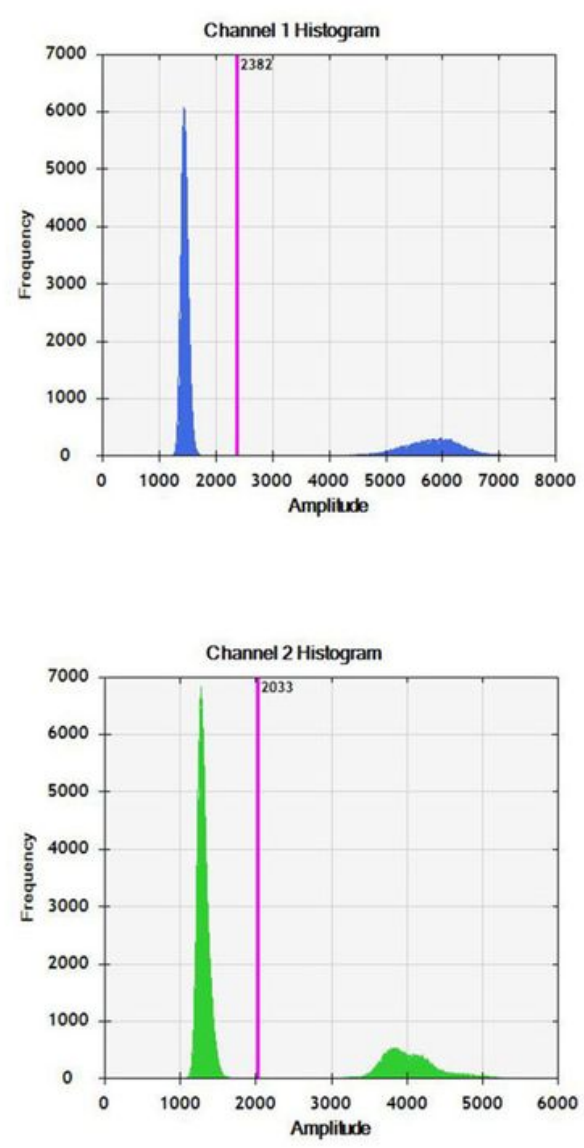

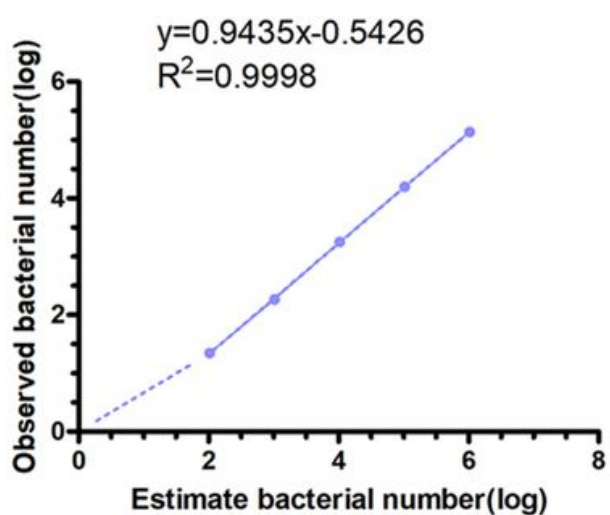

Estimate bacterial number $(\log )$

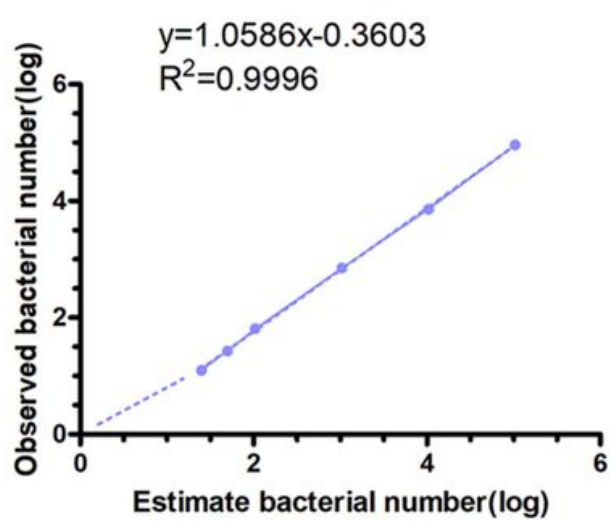

Figure 4

Detection of blood samples of simulated bacteremia blood sample by ddPCR system. A: The 1D droplet spots of FAM fluorescence for E. coli ATCC25922 were shown on the left and middle. The estimated number of Escherichia coli ATCC25922 nucleic acid template is $1.04 \times 106,1.04 \times 105,1.04 \times 104,1.04 \times 103$, $1.04 \times 102$ and NC. B: The 1D droplet spots of VIC fluorescence for Staphylococcus aureus ATCC25923 were shown on the left and middle. The estimated number of Staphylococcus aureus ATCC25923 nucleic acid template is $1.04 \times 105,1.04 \times 104,1.04 \times 103,1.04 \times 102,50,25$ and NC. The linear fitting curves of $E$. coli ATCC25922 and Staphylococcus aureus ATCC25923 were shown on the right. Error bars represent the standard deviation of three replicate samples at each target bacterial count. 


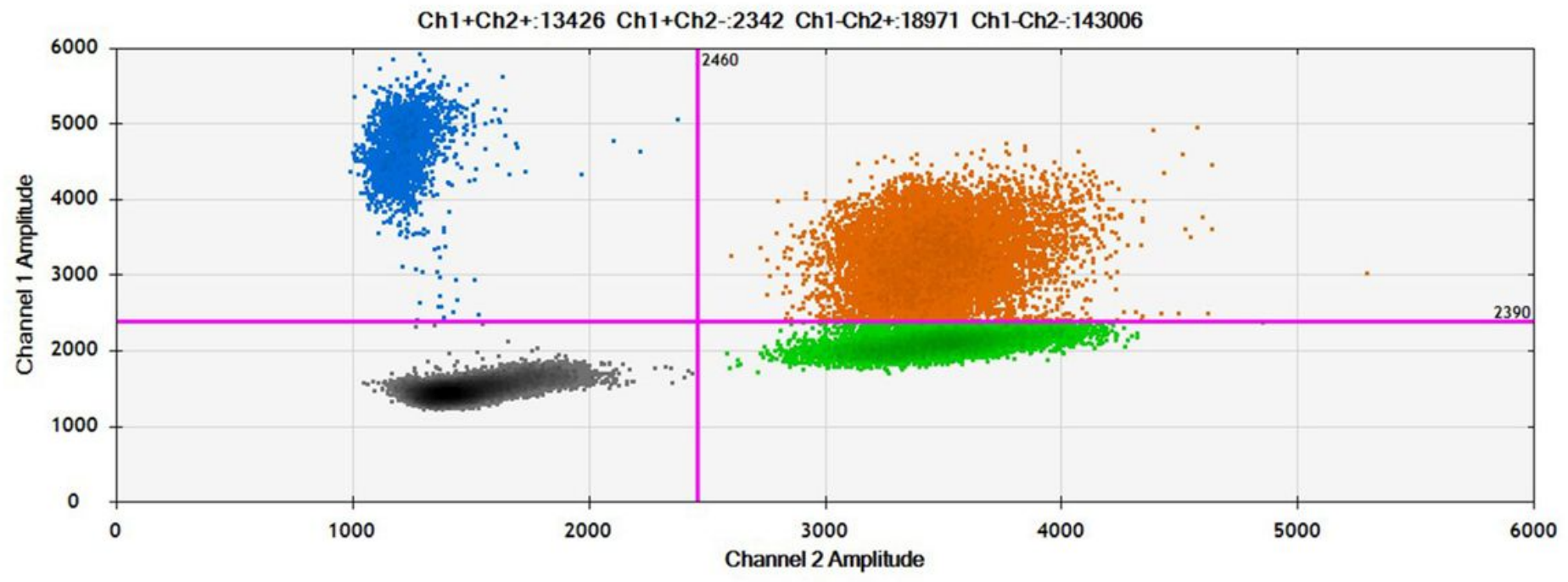

A

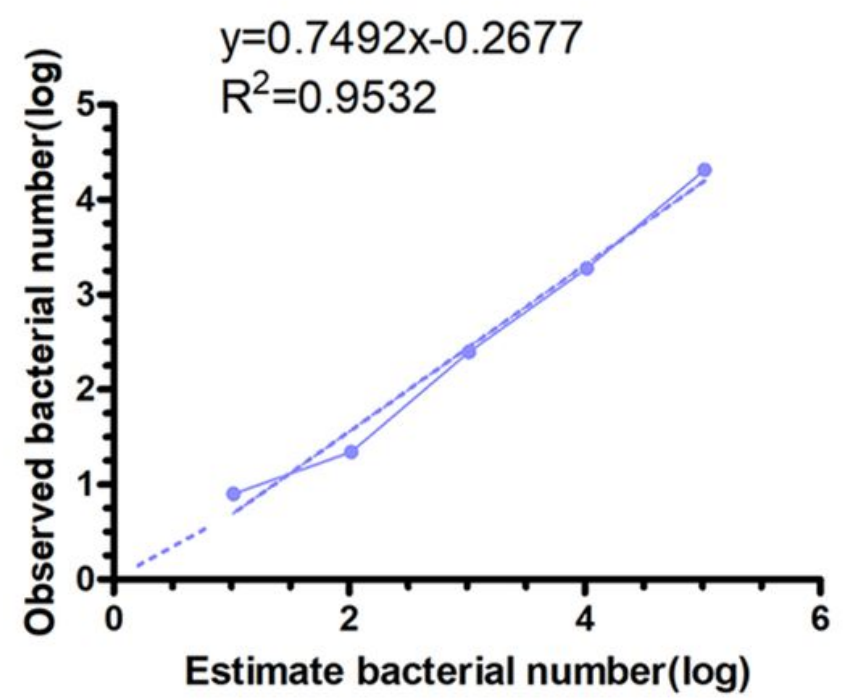

B

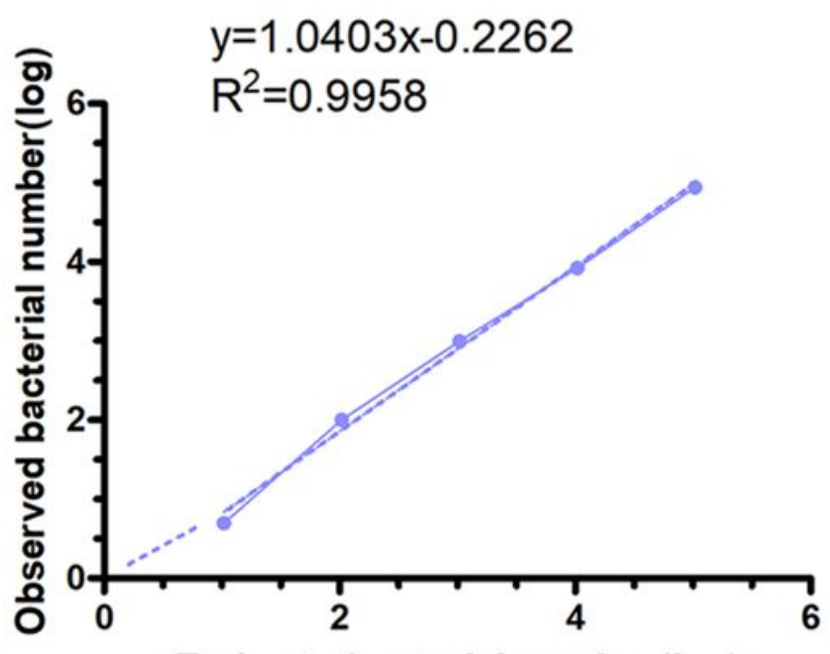

Estimate bacterial number(log)

\section{Figure 5}

Quantitative results of the ddPCR system simultaneously detecting simulated bacteremia blood sample with two bacterial strains. A: The 2D drop plots of Escherichia coli ATCC25922 and Staphylococcus aureus ATCC25923 by ddPCR system. B: The linear fitting curves of Escherichia coli ATCC25922 were shown on the left. C: The linear fitting curves of Staphylococcus aureus ATCC25923 were shown on the right.

\section{Supplementary Files}

This is a list of supplementary files associated with this preprint. Click to download. 
- FigureS1.jpg

- Figures2.jpg

- SupplementaryFigureLegend.docx

- SupplementaryTables.docx 